\title{
Memory Footprint Reduction for FPGA Routing Algorithms
}

\author{
Scott Y.L. Chin, and Steven J.E. Wilton \\ Department of Electrical and Computer Engineering \\ University of British Columbia \\ Vancouver, B.C., Canada \\ email: scottc@ece.ubc.ca, stevew@ece.ubc.ca
}

\begin{abstract}
In this paper, we present a technique to reduce the run-time memory footprint of FPGA routing algorithms. These algorithms require a representation of the physical routing resources and programmable connections on the device; this representation dominates the storage requirements of FPGA routers. We show that by taking advantage of the tile-based nature of FPGAs, we can reduce the amount of information that must be explicitly represented, leading to significant memory savings. To make our proposal concrete, we applied it to the routing algorithm in VPR and quantified the impact on run-time memory footprint, and place and route compile-time. We found that a memory reduction of $5 X$ to $13 X$ could be achieved at a routing runtime penalty of $2.26 \mathrm{X}$ and an overall place-and-route runtime penalty of $1.28 \mathrm{X}$
\end{abstract}

\section{Introduction}

Advancements in process technology have allowed for a dramatic increase in the capacity of commercial FPGA devices and this scaling is continuing at a steady pace. This places increasing demands on the accompanying FPGA CAD tools, resulting in larger run-time memory requirements, and longer compile times.

Reducing the compile time for CAD tools is an active area of research. Approaches include reducing the solution space explored [1][2], parallelization [3][4], and hardware acceleration [5]. These works generally do not consider the memory footprint of the CAD tools. Unlike a CAD tool targeting an ASIC, a CAD tool targeting an FPGA must be aware of every programmable switch and routing resource on the device. As devices grow, the memory footprint needed to store this information grows. Already, CAD tools from some vendors require 64-bit machines to map circuits to large devices due to the memory requirements of the placement and routing algorithms. Other vendor tools run on 32-bit machines, but increasing FPGA size will mean even these will soon require larger, more expensive workstations.

Although the memory capacity of workstations is constantly increasing, reducing the memory footprint of FPGA CAD tools is important for a number of reasons:
1. Power-users typically have access to modern workstations. However, there are a subset of designers that are designing circuits for FPGAs using low-cost workstations. FPGAs provide a low-cost low-risk entry to integration; this advantage is somewhat reduced if FPGA CAD tools require expensive workstations. As a concrete example, academic users performing FPGA architecture experiments often have a large bank of low-cost workstations to perform repeated place and route experiments. In such an environment, it is far more important to be able to compile a benchmark circuit with the systems available; the run-time of each experiment is secondary.

2. When a workstation has insufficient physical memory to perform a compile, it begins to use the swap memory on the hard disk. Accessing the hard disk is much slower than accessing RAM memory. If the process is constantly reading and writing memory to the hard drive (thrashing), then the perceived speed of the workstation is drastically reduced.

3. A small memory footprint may provide better cache performance, since more of the working set can be stored in the cache.

4. One of the most promising approaches to reduce compile time is to parallelize the CAD algorithms. One of the challenges with parallelization is coordinating access to memory. It may be that small data structures that can be replicated across processors is more suitable than a single large data structure that must be shared by all processors.

For all these reasons, reducing the memory footprint of FPGA CAD algorithms is important. Left unabated, the increasing memory footprint will become a hindrance to the continued scaling of FPGA devices.

One of the most memory intensive steps in the FPGA CAD flow is routing [6]. The goal of the router is to use the prefabricated routing resources to implement each connection in a circuit. This requires a map of all routing resources available on the FPGA. This map is commonly represented using a directed graph called the Routing Resource Graph (RRG). Typically, the nodes in the graph represent wires and pins on the FPGA, and edges 
represent programmable connections. As the number of programmable elements and wiring tracks increase, this graph grows very quickly in size, and tends to be a dominating factor in the overall memory footprint of the FPGA CAD flow

In this paper, we use this insight to reduce the memory footprint of an FPGA routing algorithm. The key is a new representation of the physical routing resources on an FPGA. FPGAs typically consist of a number of similar tiles, and the resources within each tile are the same. Our approach is to explicitly represent the connections with each tile type using a graph similar to an RRG, adding edges to represent connections between tile types. This allows us to significantly reduce the run-time memory footprint of the routing step, which in turn reduces the overall memory requirements of the FPGA CAD flow. This representation, however, requires changes to the search algorithm at the heart of the FPGA router; these changes increase the runtime of the routing algorithm. Thus, there is a trade-off between memory footprint and run-time.

To make our ideas concrete, we have implemented our ideas in the commonly used academic place and route tool [7], and quantified the impact on memory footprint and runtime. Our modified version of the VPR tool is available to the research community.

This paper is organized as follows. Section 2 gives background information on how physical routing information is represented in a routing resource graph. Section 3 then examines how the various detailed routing architecture parameters affect the RRG size. Section 4 presents the new routing resource graph representation and Section 5 then describes necessary changes to the routing algorithm. Section 6 reports the impact on memory footprint. Section 7 reports the impact on compile time. Finally, Section 8 comments on the impact on the routing solution.

\section{Background}

In this section, we describe how physical data is represented in an FPGA router, and show how previous researchers have attempted to reduce the storage required for this data. Although our discussions focus on the VPR router, other FPGA routers would have similar storage requirements.

\subsection{Routing Resource Graphs}

Two types of data must be maintained during routing. The first is a map of the physical segments and programmable switches in the architecture. The second is temporary data that is maintained during the routing process (in the Pathfinder algorithm, for example, this would include the occupancies, current cost, history cost, and base cost of each routing segment [8]). Some routers, such as VPR, combine this information into a single structure.

The map of the physical segments and programmable switches can be represented by a Routing Resource Graph (RRG). In the VPR router, the RRG is a directed graph where each wire and logic pin on the FPGA is represented by a node. In addition to wire and pin nodes, there are also source and sink nodes to model pins that are logically equivalent. Programmable connections between the resources are represented by directed edges. Directional connections such as tristate buffers are modeled by a single edge, and bidirectional connections such as pass transistors are modeled by a pair of edges. Figure 1 shows an example of the routing resource graph for one logic block tile containing a 2-input lookup table (LUT) and a channel width of 2 .
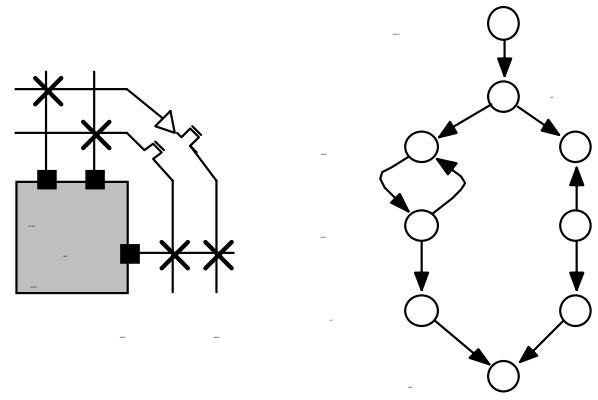

Figure 1. Example portion of the routing resource graph

In the VPR router, each node of the routing resource graph includes not only connectivity information, but also coordinates for mapping each node to its corresponding physical resource, information for the timing model (capacitance and resistance of each physical resource), and the capacity of each node. In addition, in VPR, each node contains temporary information for the router, including the current cost, history cost, and base cost of each node, the current occupancy of each node.

In the VPR router, the routing resource graph for a 200x200 FPGA with 150 tracks per channel and segment length of four requires 1.6GB of storage. Of this, the majority (1.58GB) is due to the static information that describes the connectivity, while only a small portion $(\sim 0.02 \mathrm{~GB})$ is due to the dynamic information created by the router. This motivates us to focus on reducing the storage requirements for the static information; future work will investigate whether it is possible to improve the efficiency of the dynamic information storage.

\subsection{Previous Work}

There has been only a few papers focusing on memory reduction. The most relevant proposes a Just-in-Time (JIT) compiler for FPGAs, which configures an FPGA when it is about to execute [6]. Their routing algorithm uses a simplified graph where each node represents a switch box, and edges represent routing resources between the switch boxes. Although their technique produces a significant reduction in memory footprint, it only applies to simplified architectures aimed for fast JIT compilation. 


\section{Impact of Routing Architecture}

From the previous discussion it is clear that the size of the routing resource graph depends on the detailed routing architecture. To help motivate our technique, we first measure the impact of routing architecture on the storage requirements of an existing routing algorithm.

We focus on six parameters that are used to define the detailed routing architecture in VPR. These parameters are summarized in Table 1 and are described in detail in [7].

\begin{tabular}{c|c}
\hline Symbol & Description \\
\hline \hline$N$ & Array size (NxN array) \\
$W$ & Channel Width \\
$L$ & Segmentation Length \\
$F c$ & Connection Box Population \\
Frac_sb & Switch Box Internal Population \\
Frac_cb & Connection Box Internal Population \\
\hline
\end{tabular}

Table 1. Detailed Routing Architecture Parameters

Figure 2(a) though (f) shows how the memory footprint depends on the architecture parameters. The results were obtained by routing the same circuit on different FPGA architectures using VPR. In each architecture we hold all parameters constant except the one under investigation. For parameters such as Fc, Frac_sb, Frac_cb, which affect the routability of a circuit, we choose a large $W$ so that the it can remain fixed as we vary the parameter under investigation. When varying Frac_sb and Frac_cb, we used $L=4$.

All results have been normalized; for $N$ and $W$, we normalize to the largest size used. For $L$, the results have been normalized to unit-length wires. For Fc, Frac_sb, and $F r a c \_c b$, the results are normalized to the fully populated case.

These figures show that $N, W$, and $L$ have the most significant impact on the RRG memory size. The reason is that these parameters affect the number of nodes in the graph, whereas Fc, Frac_cb, Frac_sb affect the number of edges. Since the amount of information required for a node is substantially larger than the amount required for an edge, $N, W$, and $L$ play a more dominant role. This further highlights that to reduce the memory footprint of the RRG, we need to focus on reducing the number of nodes.

\section{Proposed Technique}

From Section 2, the majority of the memory footprint in a typical router is due to the connectivity information in the static routing resource graph. In this section, we present a new representation for this information that requires significantly less storage.

Our technique takes advantage of the tiled nature of FPGAs. Rather than explicitly representing all the tracks and programmable resources in the routing resource graph, we divide our fabric into tiles. Each tile corresponds to a small number of tile types, where all tiles within a tile type are identical (with the possible exception of the starting and ending point of wires that span more than one block). For example, a tile type might contain a single logic block and

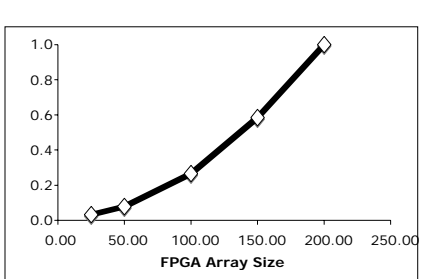

a) Array Size

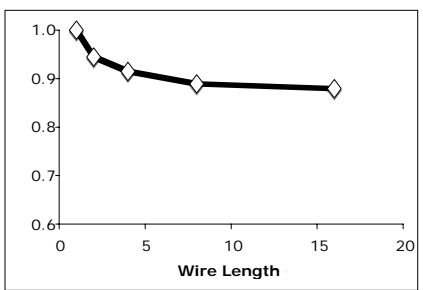

c) Wire Length

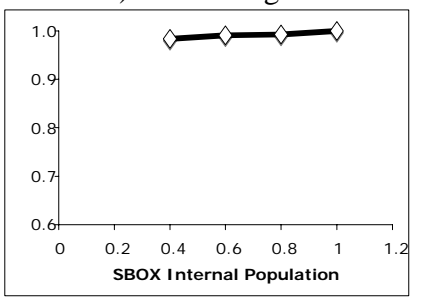

e) SBOX Internal Population

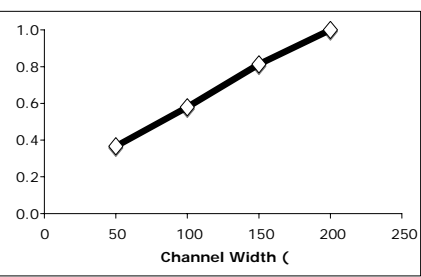

b) Channel Width

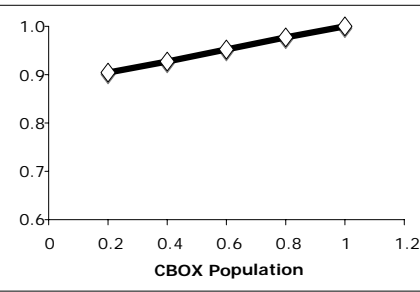

d) Cbox Population (Fc)

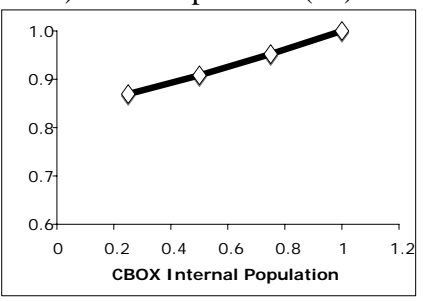

f) CBOX Internal Population
Figure 2: Impact of detailed routing architecture parameters on memory footprint.

its surrounding routing channels. An FPGA with embedded blocks would contain a tile type consisting of the embedded block and its surrounding routing. Additional tiles are required for the ring of I/O blocks.

Each tile type is modeled in detail using a graph similar to a routing resource graph. Connections between neighbouring tiles are then represented by adding additional "wrap-around" edges. Special care is taken to model wire segments that span more than one tile. The resulting graph is significantly smaller than a full routing resource graph, yet contains the exact same information.

In the following subsections, we describe our technique in more detail. This technique requires some modifications to the routing algorithm; in Section 5, we show how our proposal can be incorporated into an FPGA router.

\subsection{Tile Type Representations}

We illustrate our technique on an FPGA without embedded memories or DSP blocks. For such an FPGA, we require five tile types:CLB, IO_TOP, IO_BOTTOM, IO_LEFT, IO_RIGHT. These five tiles are shown in the first row of Figure 3 . The second row shows a representation of each tile, where each pin has been replaced by a graph node. 


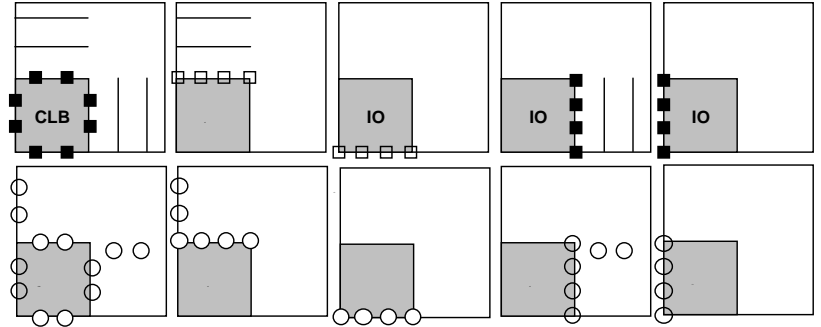

Figure 3: The five tiles required to model an FPGA consisting of logic and IO blocks

Programmable connections are modeled differently depending on whether they are between nodes in the same tile, or between nodes in different tiles. In the following three subsections, we describe each of these separately.

\subsection{Connections Within the Same Tile Type}

For programmable connections between resources that lie within the same tile instance, the edges are modeled in the traditional manner as shown in Figure 4.
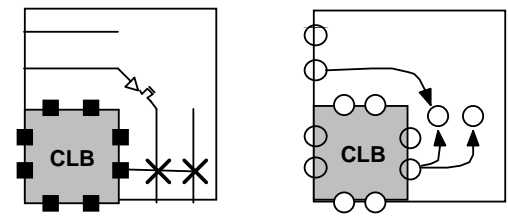

Figure 4. Connections within the same tile instance.

\subsection{Connections Within Different Tiles of the same Type}

When a programmable connection exists between routing resources that belong to different instances of the same tile type, we use an edge that "wraps around" as shown in Figure 5. The upper connection in this example is a connection between two horizontal wire segments. In our graph of the tile, both of these wire segments are represented by the same node. When this happens the node emits an edge that returns to itself. The edge is labeled with two quantities: deltax and deltay. These values specify to which adjacent tile instance the edge connects. In this example, the delta values for the edge are $(+1,0)$. All edges are labeled in this way.
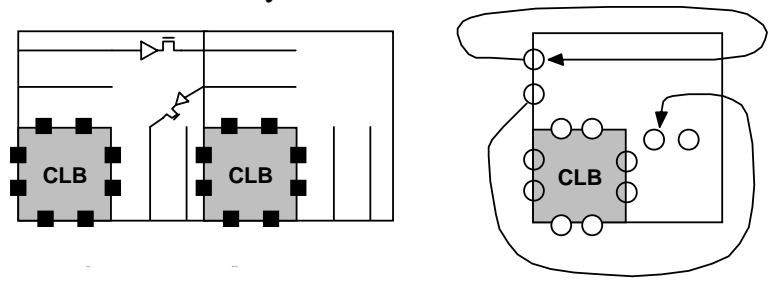

Figure 5. Connections to different instances of the same tile type.

In the lower connection in Figure 5, a horizontal wire segment connects to a vertical wire segment. In this case, the two resources are represented by different nodes but are still part of the same tile type. By specifying the (deltax,deltay) values correctly $((-1,0)$ in this example) the connectivity information is completely preserved.

\subsection{Connections Between Different Tile Types}

Programmable connections between tiles belonging to different tile types can be handled in the same way. However, sometimes the type of an edge's destination tile depends on the coordinates of the tile in the FPGA array. For example, consider a logic block tile at the centre of an FPGA. The neighbouring tiles in this case will be other logic block tiles. On the other hand, a logic block tile on the periphery of the FPGA would have some neighbours that are IO block tiles.

In this case, edges are added from the source node to all potential destination nodes in all potential tile types. This is shown graphically in Figure 6 in which two edges are sourced from the horizontal track in the right-most logic block tile. One edge wraps around to the same logic block tile, while the other edge connects to the corresponding I/O block tile type. The two edges are given the same (deltax,deltay) labels $((-1,0)$ in this case).

Clearly, in this situation, only one of the two edges corresponds to a physical routing switch for any given tile; which edge depends on the coordinates of the tile. It is up to the routing algorithm to keep track of the current $\mathrm{x}, \mathrm{y}$ location during maze routing, and only use those edges which correspond to physical switches. More details regarding the router are in Section 5.
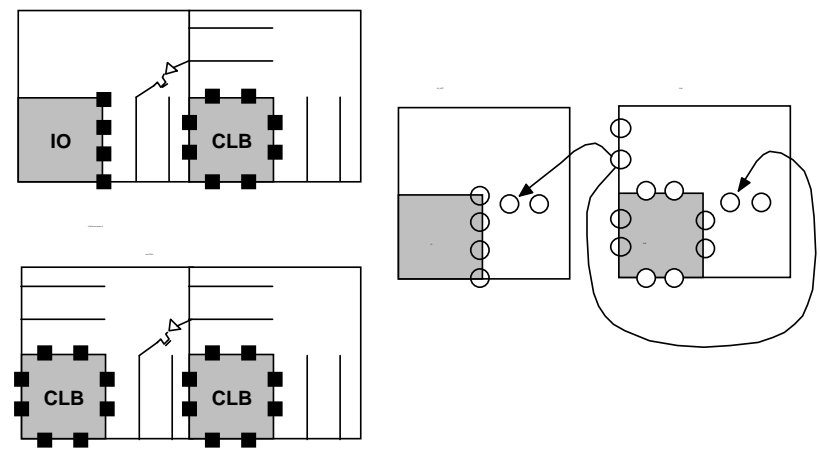

Figure 6. Instance-dependant connections

\subsection{Modelling Long Wires}

Wires that span more than one tile $(\mathrm{L}>1)$ require special attention. Consider a horizontal wire within a logic block tile. At each instance of this logic tile, the horizontal wire may end, or it may pass through to the neighbouring instance using a hard connection. We can represent this using two edges, and the choice of which edge is used by the router depends on the current $(\mathrm{x}, \mathrm{y})$ location being explored by the maze router. However, the edge that corresponds to the hard connection is different in that it does not correspond to a programmable switch. We use a special type of edge called a delayless connection to represent this. 


\subsection{Depopulated Connection and Switch Boxes}

The above has implicitly assumed that the connection blocks within each logic block tile are identical. However, as described in [7], this may not be the case; in a depopulated connection box architecture, some logic blocks may have different values of $F c$. Although this could be modeled by creating a separate tile type for each logic block with a unique connection block pattern, it is more efficient to represent all possible connection block connections and leave it to the router to determine which edges correspond to physical switches (as in Section 4.3) based on the (x,y) location that is currently being explored. Architectures with depopulated switch blocks (in which not every switch block contains connections to all tracks) can be handled in the same manner.

\section{Impact on the Routing Tool}

The above representation places new demands on the routing algorithm. In this section, we show how an existing routing algorithm, the one employed in VPR, can be modified to work with our new representation.

\subsection{Routing Related Information}

Section 4 showed how the storage required to represent the connectivity information of an FPGA can be reduced significantly. However, as indicated in Section 2, the router also must maintain temporary information to aid in routing. In the Pathfinder algorithm, for example, the occupancy, current cost, base cost, and history cost of each node in the routing resource graph needs to be maintained. This information can not be compressed as easily as the connectivity information, since different tracks in each tile may be used to implement different nets. Thus, we maintain separate storage for these quantities and do not compress this storage along with the routing resource graph. This is different than the VPR router, which stores these quantities in the same structure as the routing resource graph.

\subsection{Changes to the Routing Algorithm}

In general, the router has two new tasks:

1. To keep track of the $(x, y)$ coordinates of each routing resource that is visited during maze expansion.

2. To determine which edges should be traversed during expansion.

Figure 7 shows the pseudo-code for the Pathfinder-based VPR router. As long as the sink has not been found, the node with the best score is removed from the priority queue (PQ) and its neighbouring nodes are added back into the PQ. Since the router always knows the $(x, y)$ coordinates of the source and sinks being routed, the $(x, y)$ coordinates of every subsequent node that is added to the $P Q$ can be calculated using the $(\mathrm{x}, \mathrm{y})$ coordinate of the source node as the starting point, and the relative positioning information stored in the (deltax, deltay) edge labels.

When expanding the neighbours of a node, the router iterates across the node's list of fanout edges and adds each and every node to the PQ. We modify this to handle nodes representing wire segments that are longer than unit length (i.e. $L>1$ ) and to check which edges should be used for expansion. The modified pseudo-code is shown in Figure 8. This code will be further illustrated in an example in Section 5.3.

As described in Sections 4.3 to 4.5, not all edges in the graph correspond to physical switches. The routine fanout_exists uses the $(\mathrm{x}, \mathrm{y})$ coordinates of the tile being explored to compute whether each edge corresponds to a physical switch. The details of these calculations are not shown, but they are straightforward given the detailed routing architecture of the fabric being modeled.

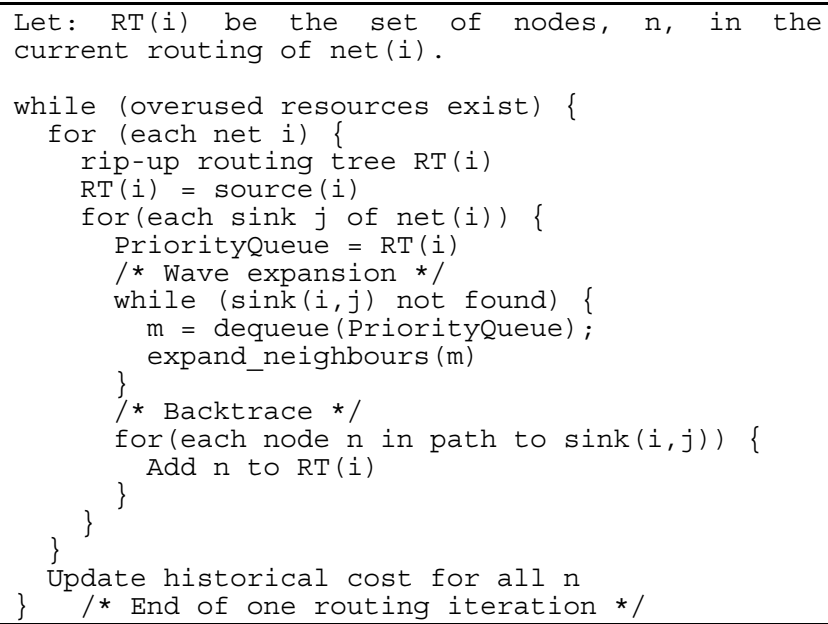

Figure 7: Pseudo code for the VPR router.

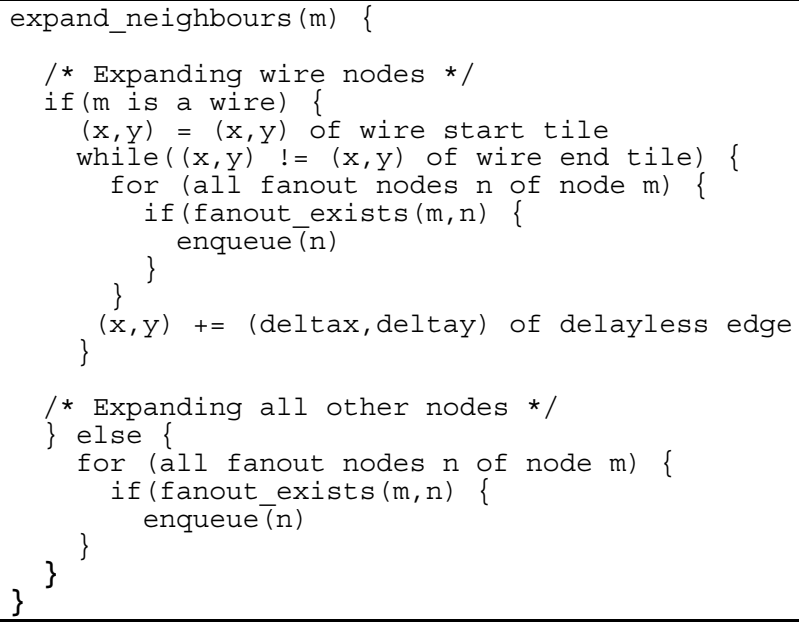

Figure 8. Pseudo-code highlighting changes to the steps performed during neighbour expansion.

\subsection{Example Illustrating Long Wires}

This subsection presents an example to illustrate how long wires are handled by the algorithm. Figure 9 shows a part of an FPGA with a horizontal wire segment with $L=4$. The wire has a Frac_sb=0.6 meaning that it has an SBOX connection at its end points and at one internal tile. In the original algorithm, a single node represents this 
wire and the node has seven fanout edges. Using our representation, the router must go through several steps to determine the appropriate fanout edges of the wire segment. First it determines the starting $(\mathrm{x}, \mathrm{y})$ location of the wire segment. This can be calculated given the track number and the $(x, y)$ position of any part of the wire. Next, the router traverses the delayless connection edge until it reaches the end point tile instance. The $(\mathrm{x}, \mathrm{y})$ position of the end point tile can be calculated in a the same way as the start point. Each time the router traverses the delayless connection, it has three potential edges that it can expand: an edge to the CLB pin, an edge to the vertical wire node within the same tile instance (denoted as edge A), and an edge connecting to the vertical wire node that is one tile instance to the left (denoted as edge $B$ ). In this example, edge $B$ would be expanded only in the starting tile, and edge A would only be expanded in the second and last tiles. The pin edge is expanded in all iterations and the delayless edge is traversed three times.
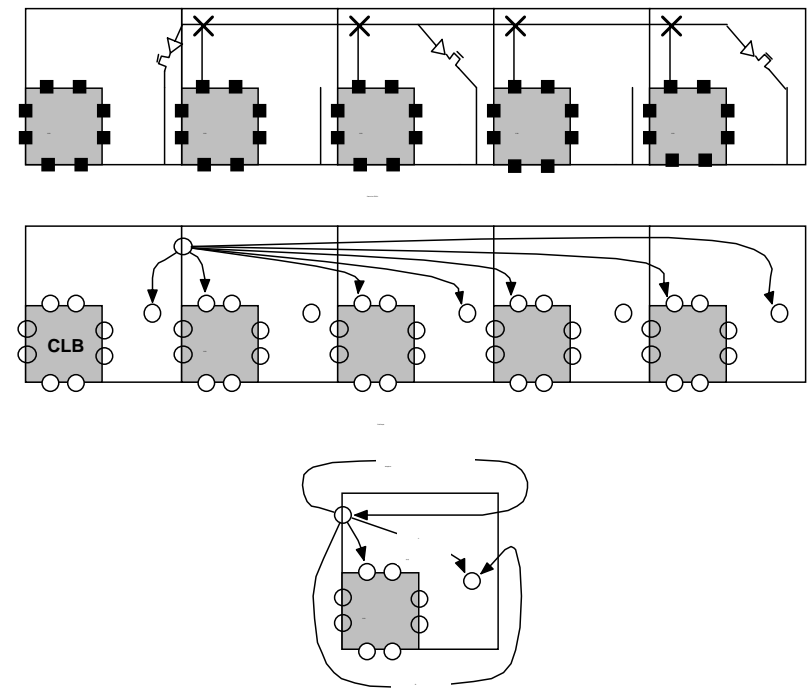

Figure 9.Example for how the router expands long wires

\section{Results}

In this section, we measure the memory footprint savings obtained by our proposal.

\subsection{Experimental Framework}

In each experiment, we pause the VPR process after it has loaded all of the data structures required for routing, but before the routing algorithm actually starts. We then measure the virtual memory usage of the VPR process. Virtual memory is the amount of memory that the process requires regardless of how much physical memory is actually installed on the machine. We take this measurement using the UNIX command top. Top's ability to give detailed memory usage information is sufficient for our purpose.
In the following experiments, we route the same circuit to a number of different architectures that vary in $N, W$, and $L$. For each architecture, we assume that logic blocks have 22 inputs and consists of ten 4-input LUTs and registers.

\subsection{Experimental Results for Array Size $\boldsymbol{N}$}

Figure 10 shows the memory requirements of our router and the original VPR router as a function of the array size N. For small array sizes (i.e. $\mathrm{N}=25$ ), the memory footprint of the proposed technique is only slightly better (1.7x smaller). As the array size increases, the difference in memory footprint becomes more dramatic. At $\mathrm{N}=200$, the memory footprint of the proposed technique is $6.5 \mathrm{x}$ smaller. Note that a 200x200 architecture with a channel width of $\mathrm{W}=150$ is comparable to current commercial devices [9] [10].

Although the storage required for the routing resource graph information is no longer a function of $\mathrm{N}$, the storage required to implement the temporary routing structures described in Section 5.1 is still proportional to $\mathrm{N}$. This explains why our storage requirements grow slightly as $\mathrm{N}$ increases.

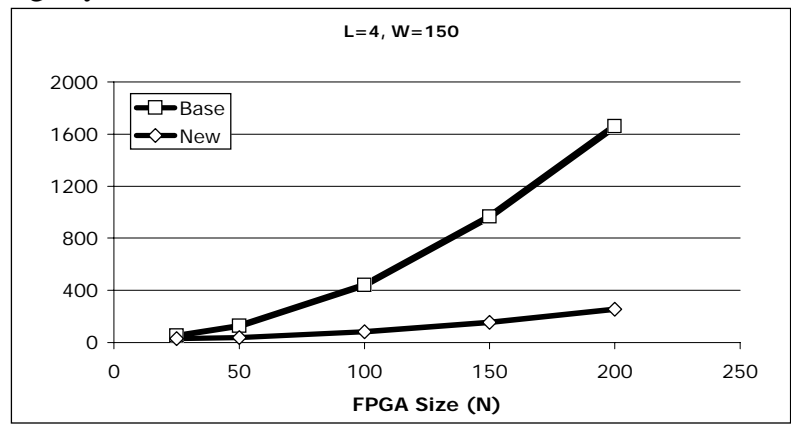

Figure 10. Memory footprint of VPR using the original and proposed RRG representations. $\mathrm{W}=150, \mathrm{~L}=4$.

Figure 11 shows how the memory savings depends on $\mathrm{N}$ for various fixed channel widths. The vertical axis is the baseline memory footprint / new memory footprint (this is the memory savings). Each line corresponds to a different channel width. The wire length is $\mathrm{L}=8$ (we also looked at $\mathrm{L}=1, \mathrm{~L}=4$, and $\mathrm{L} 16$ and the conclusions were similar). As shown in the graph, as $N$ increases, the memory savings increases but reaches an asymptote.

The asymptote can be explained as follows. Consider the original representation. Assume the storage requirements for the connectivity information and temporary routing information to grow at a rate of $c^{*} \mathrm{~N}^{2}$ and $\mathrm{r}^{*} \mathrm{~N}^{2}$, respectively, where $c$ and $r$ are constants. Hence, the overall footprint grows at $(\mathrm{c}+\mathrm{r})^{*} \mathrm{~N}^{2}$. In the new representation, denote the storage requirements for the connectivity and temporary routing information to grow at a rate of $\mathrm{C}$ and $\mathrm{r}^{*} \mathrm{~N}^{2}$ where $C$ and $r$ are also constants (the storage requirements for the connectivity information is not a function of $\mathrm{N}$ in the new representation). This gives an overall growth rate of 
$\mathrm{C}+\mathrm{r}^{*} \mathrm{~N}^{2}$. Note that $r$ is the same constant in both representations because the routing information is not compressed in the proposed technique.

Using these definitions, the memory reduction factor is $\left((\mathrm{c}+\mathrm{r}) * \mathrm{~N}^{2}\right) /\left(\mathrm{C}+\mathrm{r}^{*} \mathrm{~N}^{2}\right)$. When $\mathrm{N}$ becomes large, $C$ becomes insignificant and the ratio approaches $(\mathrm{c} / \mathrm{r})+1$. This value becomes the asymptote for the amount of memory that can be saved for the family of architectures with a given $W$ and $L$.

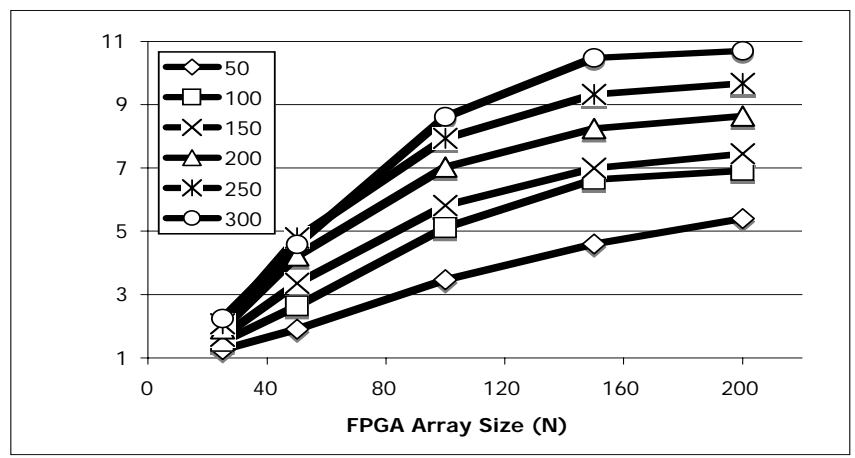

Figures 11 . Memory reductions for $\mathrm{L}=8$ across various values of $\mathrm{N}$ and $\mathrm{W}$.

By using the case of $\mathrm{N}=200$, we can conservatively approximate the asymptote. Figure 12 shows this approximation where each line represents a different wire length. This figure shows that for small channel widths $(\mathrm{W}=50)$, we get approximately a $3 \mathrm{x}-6 \mathrm{x}$ reduction in memory footprint. For larger channel widths, we can get approximately $5 \mathrm{x}-13 \mathrm{x}$ reduction depending on $\mathrm{L}$.

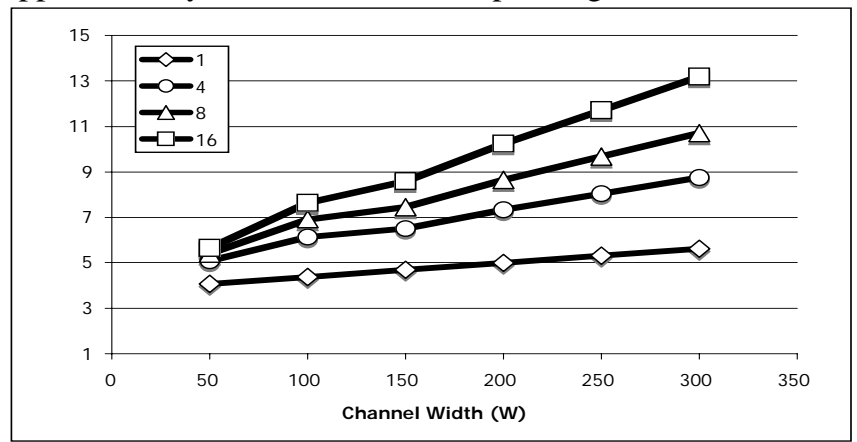

Figure 12. Memory reductions for $\mathrm{N}=200$ across various values of $\mathrm{L}$ and $\mathrm{W}$.

\subsection{Experimental Results for Channel Width}

Figure 13 shows the storage requirements as a function of channel width for a family of architectures with $\mathrm{N}=100$, and $\mathrm{L}=4$. The storage requirements of both representations increase linearly but differ by a constant factor. This is due to the absence of the dependance on $N$ in the new representation. As an example, suppose we increase $W$ by 1. In the CLB tile of the proposed technique, the number of nodes is increased by two (one for the horizontal channel and one for the vertical channel). But using the original representation, the number of nodes for CLB channels is increased by $\mathrm{N}^{*} 2$ because there are $N$ CLB tiles.

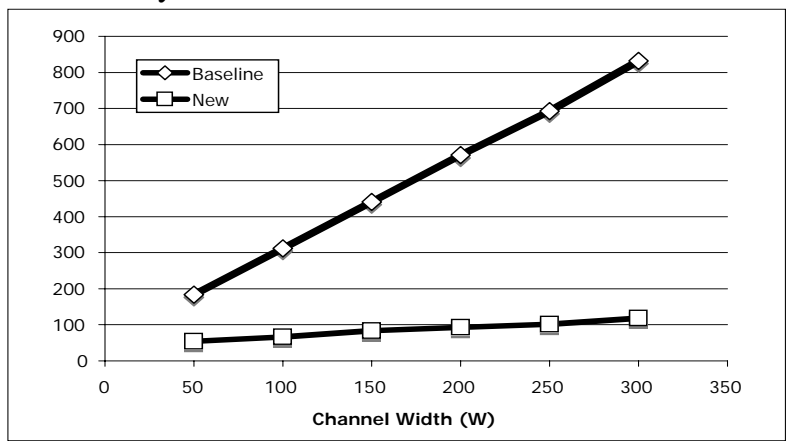

Figure 13. Memory footprint of VPR using the original and new representations. $\mathrm{N}=100, \mathrm{~L}=4$.

\subsection{Experimental Results for Wire Length}

Figure 14 shows that the memory reduction also changes with L. As L increases, less storage is required for wire resources. This reduces both the connectivity and temporary information in the original representation. In the proposed technique, this only reduces the temporary information. Since the connectivity information in the proposed technique is fixed and relatively small, its overall footprint decreases much faster than the full RRG, leading to an overall increase in memory reduction.

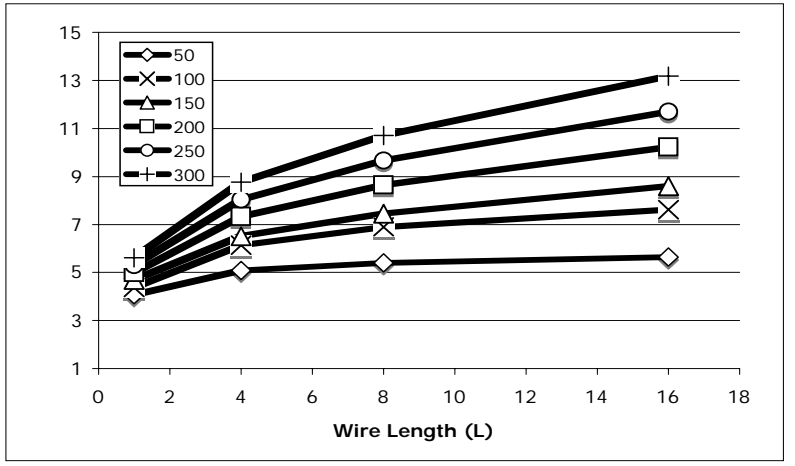

Figure 14. Memory reductions for $\mathrm{N}=200$, acroos various values of $\mathrm{W}$ and $\mathrm{L}$.

\section{Impact on Compile Time}

The additional steps added to the router described in Section 4 will increase the router's execution time. In this section, we measure this increase.

Both versions of VPR were instrumented to count the number of executed clock ticks at three points in the flow: immediately before placement, after placement, and after routing. These three values allow us to calculate the placement time, routing time, and total compile time. All measurements were performed on an Intel Core2 Duo $1.87 \mathrm{GHz}$ processor. 


\begin{tabular}{|c|c|c|c|c|c|c|c|c|c|}
\hline \multirow[t]{2}{*}{ Benchmarks } & \multirow[t]{2}{*}{$\mathrm{N}$} & \multirow[t]{2}{*}{$\bar{W}$} & \multirow{2}{*}{$\begin{array}{l}\text { Placement } \\
\text { Time }\end{array}$} & \multicolumn{2}{|c|}{ Baseline } & \multicolumn{2}{|c|}{ New } & \multicolumn{2}{|c|}{ Ratio } \\
\hline & & & & Route Time & Total Time & Route Time & Total Time & Route Time & Total Time \\
\hline 30seq20com & 43 & 72 & 321.07 & 100.16 & 421.23 & 224.88 & 545.95 & 2.25 & 1.30 \\
\hline $3 \operatorname{lrg} 50 \mathrm{sml}$ & 35 & 56 & 171.14 & 44.48 & 215.62 & 103.19 & 274.33 & 2.32 & 1.27 \\
\hline 50mixedsize & 44 & 54 & 298.85 & 59.73 & 358.68 & 134.67 & 433.62 & 2.25 & 1.21 \\
\hline 60mixedsize & 43 & 62 & 312.91 & 92.29 & 405.20 & 208.52 & 521.43 & 2.26 & 1.29 \\
\hline $70 s 1423$ & 40 & 47 & 205.47 & 68.18 & 273.65 & 151.28 & 356.75 & 2.22 & 1.30 \\
\hline Lotsaffs2 & 33 & 59 & 131.49 & 29.97 & 161.46 & 69.95 & 201.44 & 2.33 & 1.25 \\
\hline $70 \mathrm{sml}$ & 33 & 99 & 198.79 & 89.04 & 287.83 & 194.88 & 393.67 & 2.19 & 1.37 \\
\hline Average & & & & & & & & 2.26 & 1.28 \\
\hline
\end{tabular}

Table 2. Compile time results for synthetic benchmark circuits

We are interested in how the proposed technique performs across different benchmark circuits because the number of nets and sinks affect the amount of time spent on routing. We use two suites of benchmark circuits. The first suite consists of the twenty largest MCNC circuits. The second suite consist of seven synthetic circuits created by stitching together a number of MCNC circuits using the technique described in [11]. Although these circuits are much larger than the twenty largest MCNC circuits, they are still not as big as some of the architectures that we investigated in Section 5. However, we found the impact on compile-time to be generally independent of circuit size.

We assume an architecture that uses length 4 wires, and logic blocks with 22 inputs and ten 4-input LUTs. Each circuit is placed and routed to the minimum array size and channel width.

Table 2 reports the compile time results for the suite of synthetic circuits. Columns 2-3 shows the minimum array size and channel width required to place and route the circuit. Columns 4-6 show the placement, routing, and overall run-times when using the original version of VPR. Columns 7-8 shows the new routing and overall run-times and Columns 9-10 shows the ratios. The average increase in routing time is $2.26 \mathrm{x}$ and the average increase in overall compile time is 1.28x. An important observation is that the overhead for each circuit is close to the average, meaning that the compile time overhead is generally independent of the FPGA size.

The average increase in route time and overall compile time for the MCNC suite were found to be 2.16x and 1.29x respectively. The detailed results for this suite have been omitted due to space but yielded the same conclusions.

\section{Impact on Routing Solution}

An important property of this technique is that all of the information in the routing resource graph is maintained exactly. This means that there is no change to the routing solution. This was verified by comparing the routing serial number generated by VPR.

\section{Conclusion}

This paper proposed a method to compress the connectivity information within the routing resource graph by taking advantage of the regularity in the FPGA. By doing so, we showed that the memory footprint of the routing step, one of the most memory intensive steps in the CAD flow, can be dramatically reduced. Due to the extra steps carried out by the router, we found that the overall place and route compile time increased on average by $1.28 \mathrm{x}$. Most importantly, the proposed technique stores the architecture information exactly.

This paper has presented an important first step towards tackling the issue of increasing memory footprint of FPGA CAD tools. Code optimizations may delay the problem somewhat, but a long-term solution requires significant changes in CAD algorithms or FPGA architectures. Finally, we have made our modified version of VPR available to the research community at http://www.ece.ubc.ca/ scottc/downloads.html. This work was supported by Altera and the National Sciences and Engineering Research Council of Canada.

\section{References}

[1] Y. Sankar, J. Rose, "Trading Quality for Compile Time: Ultra-Fast Placements for FPGAs,” Int. Symp. On Field-Programmable Gate Arrays, 1999, pp157-164

[2] C. Mulpuri, S. Hauck, "Runtime and quality tradeoffs in FPGA placement and routing," Int. Symp. on Field-Programmable Gate Arrays, 2001

[3] P.K. Chan, M.D.F Schlag, C. Ebeling, L. McMurchie, "DistributedMemory Parallel Routing For Field-Programmable Gate Arrays,” Trans. On CAD, Aug 2000, pp850-862

[4] M. Haldar, A. Nayak, A. Choudhary, P. Banerjee, "Parallel Algorithms for FPGA Placement," Proceedings of Great Lakes Symposium on VLSI, 2000, pp86-94

[5] A. DeHon, R. Huang, and J. Wawrzynek, "Hardware-Assisted Fast Routing,” Symp. On Field-Programmable Custom Computing Machines, April 2002, pp205-215

[6] R. Lysecky, F. Vahid, S. Tan, “Dynamic FPGA Routing for Just-inTime FPGA Compilation," Proceedings of Design Automation Conference, 2004, pp954-959

[7] V. Betz, J. Rose, and A. Marquardt, Architecture and CAD For Deep-Submicron FPGAs, Kluwer Academic Publishers, 1999.

[8] L. McMurchie, C. Ebeling, "Pathfinder: A Negotiation-Based Performance-Driven Router for FPGAs," Int. Symp on FieldProgrammable Gate Arrays, 1995, pp. 111 - 117.

[9] D. Lewis et. al., “ The Stratix II Logic And Routing Architecture,” Int. Symposium on FPGAs, 2005, pp14-20

[10] Xilinx, Virtex-5 User Guide, Feb 2007

[11] J. Lamoureux, S.J.E. Wilton, "Clock-Aware Placement for FPGAs,” to appear Int. Conf. on Field Programmable Logic, Aug. 2007 